\title{
Thermal-optic tuning cascaded double ring optical sensor based on wavelength interrogation
}

\author{
Yanlu Wang (王彦禄) ${ }^{1}$, Zhiping Yang (杨志平 $)^{1}$, Mingyu Li (李明宇 $)^{1 *}$, Jian-Jun He (何建军) ${ }^{2}$, and Qiushun Li (李秋顺) ${ }^{3}$ \\ ${ }^{1}$ Department of Optical Engineering, School of Opto-Electronic Engineering, Changchun University of Science and Technology, Changchun 130022, China \\ ${ }^{2}$ State Key Laboratory of Modern Optical Instrumentation, Centre for Integrated Optoelectronics, Department of Optical Engineering, Zhejiang University, \\ Hangzhou 310027, China \\ 3 Jinan GK Medtech Science and Technology Development Co., Ltd., Jinan 250000, China
}

*Corresponding author: limingyu@cust.edu.cn

Received June 12, 2021 | Accepted July 7, 2021 | Posted Online September 18, 2021

\begin{abstract}
Based on the Vernier effect of the cascaded double ring resonator (CDRR) sensor, a sensor consisting of a microfluidic control system, a sensing ring, and a reference ring with a micro-heater for thermal tuning is proposed in this paper. In wavelength interrogation, a broadband spectrometer or a large tunable range laser is not required. The shift of the output spectrum caused by the refractive index change of the sample is converted into the change of the electric power by the thermal tuning heater. Due to the Vernier effect, the sensitivity of the sensor is 20 times higher than that of the single ring. The spectral envelope of the thermal-optic tuning of the sensor was fitted by a Gaussian function in the wavelength range of $8 \mathrm{~nm}$. The experimental results showed that the sensitivity was $33.703 \mathrm{~W} / \mathrm{RIU}$, and the limit of detection was $1.34 \times 10^{-5} \mathrm{RIU}$.
\end{abstract}

Keywords: cascaded double ring; optical resonators; Vernier effect; thermal-optic effect.

DOI: 10.3788/COL202220.011301

\section{Introduction}

The integrated optical waveguide sensor based on silicon on insulator (SOI) material has the advantages of high refractive index (RI) contrast, compatibility with complementary metal oxide semiconductor (CMOS) technology, and large thermooptical coefficient of $1.84 \times 10^{-4} \mathrm{~K}^{-1}$ at $1550 \mathrm{~nm}$ of $\mathrm{Si}^{[1-4]}$. With the temperature change, the effective RI of the Si waveguide will also change, resulting in the output power and spectrum of the cascaded double ring to shift ${ }^{[5]}$. By converting the wavelength shift amount into the electric energy change, the electric energy change can be directly used for sensing.

Micro-ring resonators are widely used in optical filters ${ }^{[6-8]}$, micro-ring lasers ${ }^{[9,10]}$, optical switches ${ }^{[11]}$, and micro-ring sensors $^{[12-17]}$ due to their high-quality factor ( $Q$ factor), high sensitivity, and easy integration. In recent years, based on the research on micro-ring resonators, the sensitivity and output power of optical biosensors based on micro-ring resonators have been improved by the Vernier effect ${ }^{[18,19]}$. Sensing systems based on cascaded micro-ring resonators need to pay attention to the measurement of wavelength parameters. For wavelength interrogation sensors, a large bandwidth spectrometer and a large range tunable laser are required due to the magnification.

In this paper, the reference ring of the cascaded double-ring resonator (CDRR) sensor with a micro-heater and a microfluid control system for sensing by converting the wavelength shift into the electrical power change is investigated. The RI sensitivity will not change over the different temperatures. The RI change of the sample in the sensing ring results in the envelope peak shift of the CDRR sensor. When the temperature of the reference ring is controlled by adjusting the electric power of the micro-heater, the envelope peak of the CDRR sensor can move back to the initial position. When the RI of the measured sample is changed, the envelope of the sensor's transmission spectrum can be retrieved within an $8 \mathrm{~nm}$ wavelength measurement range by changing the electrode power of the microheater. A large-bandwidth spectrometer or a large wavelength range tunable laser is not required, which reduces the cost of the sensing system. The experimental results show that the sensitivity is $33.703 \mathrm{~W} / \mathrm{RIU}$.

\section{Theoretical Analysis}

The schematic diagram of the CDRR sensor is shown in Fig. 1. The sensor is composed of a reference ring with a micro-heater and a sensing ring from removing the up-cladding layer. The transmission of the through port for reference ring is ${ }^{[20]}$

$$
T_{r}=10 \times \log _{10}\left|\frac{k_{1} k_{2} \exp \left[-j\left(\beta-j \alpha_{R}\right) \pi R\right]}{1-t_{1} t_{2} \exp \left[-j\left(\beta-j \alpha_{R}\right) 2 \pi R\right]}\right|^{2},
$$




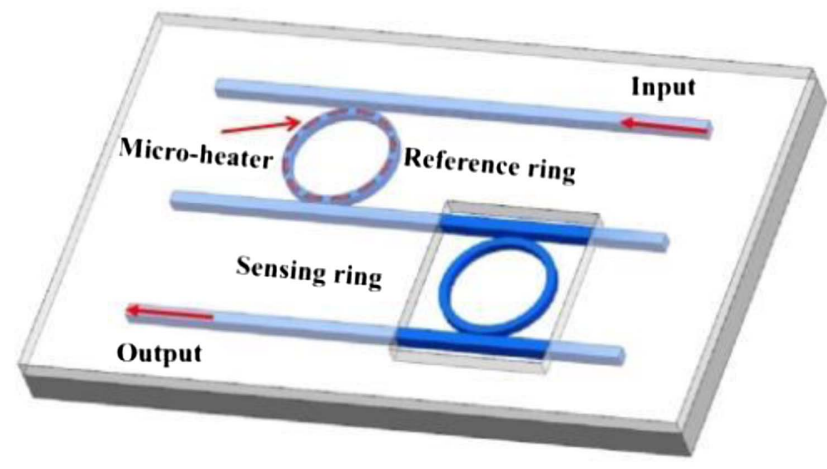

Fig. 1. Cascaded double-ring resonator sensor schematic.

where $k_{1}$ and $k_{2}$ are the coupling coefficients of the two different coupling regions of the reference ring, $t_{1}$ and $t_{2}$ are the transmission coefficients of the two different coupling regions, $\alpha_{R}$ and $\beta$ are the transmission loss and propagation constant of the reference ring, respectively, and $R$ is the radius of the reference ring.

The transmission spectrum of the sensing ring Ts and the transmission spectrum of the reference ring $T r$ have the same form, and the total transmission of the CDRR is

$$
T=T_{r} \times T_{s}
$$

When light is transmitted in a waveguide, the proportion of the evanescent wave field in the entire mode field distribution has a greater impact on the sensing performance of the device. The device adopts a strip waveguide structure, which supports the transverse electric (TE) mode and transverse magnetic (TM) mode of the two polarization modes, where the difference is that most of the energy of the TE mode electric field is in the $x$ component, and most of the energy of the TM mode electric field is in the $y$ component. The waveguide is designed with $550 \mathrm{~nm}$ width and $220 \mathrm{~nm}$ height. The TE and TM mode profiles of the waveguide are shown in Fig. 2. It can be seen that most of the energy for the TE mode is confined in the waveguide core, while most of the energy for the TM mode leaks to surrounding media. Thus, the sensitivity of the TM mode is higher than that of the TE mode.

The radii of the reference ring and the sensing ring are different, causing the $\mathrm{Tr}$ and Ts free spectral ranges (FSRs) to be unequal, as shown in Fig. 3(a). The sensor transmission spectrum envelopes change with the RI of the sample, and the electric
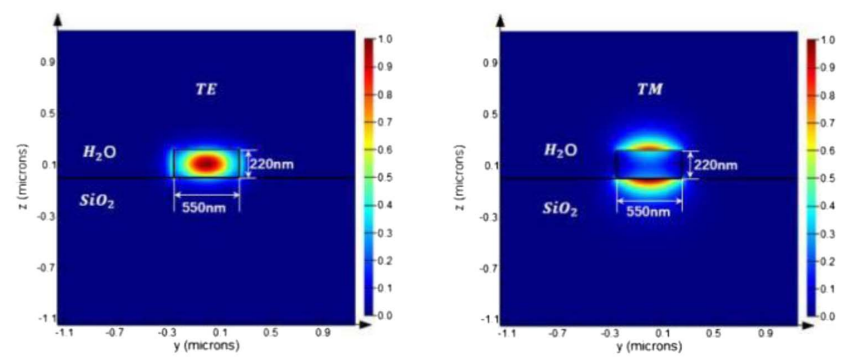

Fig. 2. Mode profiles in the Si waveguide $(550 \mathrm{~nm} \times 220 \mathrm{~nm})$.

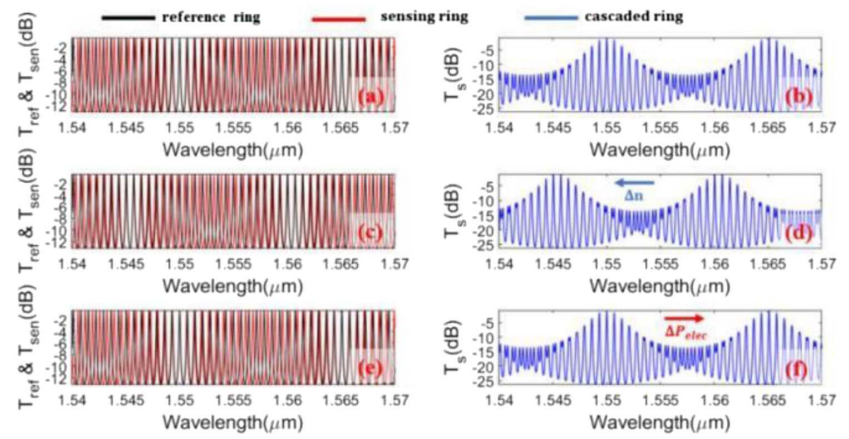

Fig. 3. Principle of thermo-optical tuning.

power variations of the micro-heater are shown in Fig. 3(b). The operating principle of the thermo-optical effect sensing system is that when the RI of the measured sample changes $\Delta n$, the drop port of the CDRR spectrum envelope shifts $\Delta \lambda$, as shown in Figs. 3(b)-3(d). At this time, the sensitivity of the CDRR is $S=\Delta \lambda / \Delta n$. When the electrical power of the micro-heater changes $\Delta P_{\text {elec }}$, the envelope of the transmission spectrum is retrieved to the original envelope, as shown in Figs. 3(b) and 3(f). The thermal tuning efficiency is $S=\Delta W / \Delta n$. The relationship between the resonance peak position of the envelope and voltage can be expressed as ${ }^{[3]}$

$$
\lambda=\sigma W+\omega=\sigma \frac{V^{2}}{R}+\omega,
$$

where $\sigma$ is the thermo-optical coefficient under wavelength interrogation, $R$ is the micro-heater resistance, and $\omega$ is a constant value.

When Fig. 3(b) overlaps with Fig. 3(f), the electrical power of the micro-heater for the retrieved spectrum envelope is determined, and the sensitivity of the sensor can be expressed as

$$
S=\Delta P_{\text {elec }} / \Delta n
$$

The shift of the transmission spectrum envelope is

$$
\partial \lambda=\sigma \partial W=2 \sigma \frac{V \partial V}{R} .
$$

It can be seen from Eq. (5) that the detection limitation is related to the accuracy of the current source voltage. Thus, the sensitivity and detection limitation can be improved by a high-precision electrical source.

\section{Experiment}

The sensing chip was based on an $8 \mathrm{inch}(1 \mathrm{inch}=2.54 \mathrm{~cm})$. SOI wafer with 220 -nm-thick top Si layer and $2-\mu \mathrm{m}$-thick buried oxide layer. The whole fabrication process compatible with the CMOS process was completed in the Integrated Circuit Advanced Process Center (ICAC) of the Institute of Microelectronics of Chinese Academy of Science (IMECAS), 


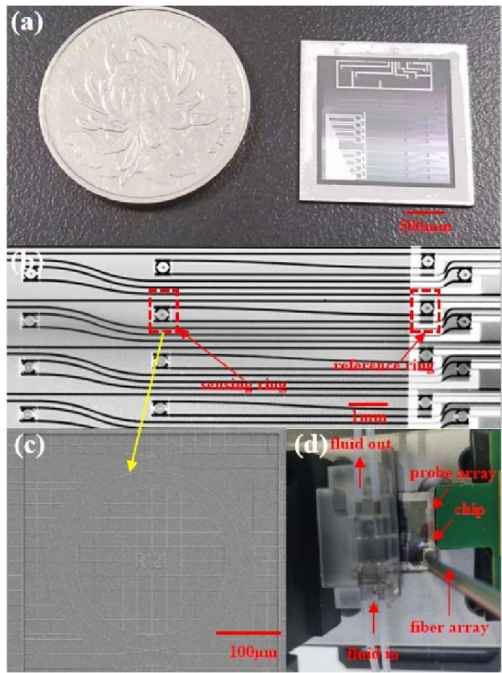

Fig. 4. (a) Photograph of the chip. (b) Optical microscope image of the CDRR. (c) SEM image of the sensing ring. (d) Photograph of the microfluidic control system.

Beijing, China. The patterns of the waveguides, grating couplers, and micro-rings were formed by the stepper and inductively coupled plasma (ICP) etching, then by growing $2 \mu \mathrm{m} \mathrm{SiO}{ }_{2}$ upper cladding by plasma enhanced chemical vapor deposition (PECVD), and the sensing window was opened by dry and wet etching. Finally, the resistance heating metal TiN and the conductive electrode $\mathrm{Al}$ layer are coated by the sputtering. The physical image of the sensor chip is shown in Fig. 4(a), and the size of the entire chip is $2 \mathrm{~cm} \times 2 \mathrm{~cm}$. The optical microscope image of the CDRR is shown in Fig. 4(b), and the SEM image of the sensing ring is shown in Fig. 4(c). As shown in Fig. 4(d), the sensing ring is removed in the upper cladding layers and combined with a microfluidic control. The liquid to be measured flows through the sensing ring, causing the RI change, resulting in spectral shift.

Figure 5 is the schematic of testing the CDRR sensor system. The tunable laser (Keysight $81606 \mathrm{~B}$ ) was controlled by Keysight Photonic Suite N7700. The laser was coupled into and out of the sensor chip by the grating couplers and was finally detected by

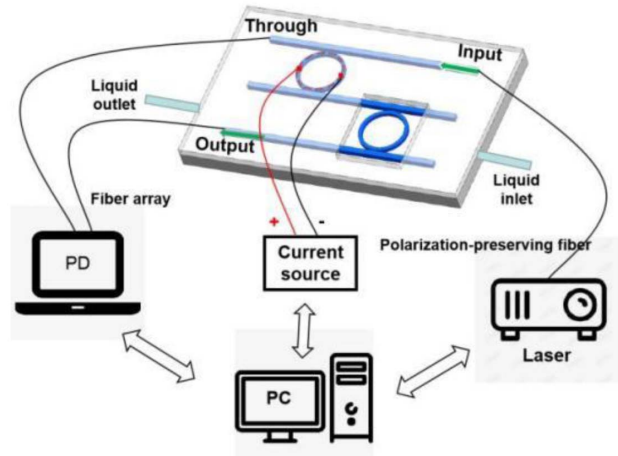

Fig. 5. Experimental device diagram. an optical power meter (Agilent $81634 \mathrm{~A}$ ). A personal computer (PC)-controlled current source (KEITHLEY 2400) is used to add electrical power on the micro-heater of the reference ring. The $\mathrm{NaCl}$ solution with different concentration was pumped into the sensing ring through the microfluidic channel. The transmission spectral envelopes for the different concentrations of $\mathrm{NaCl}$ solution were fitted ${ }^{[20]}$ in order to obtain the shift of the main resonance peak of the envelope and then obtain the sensitivity of the CDRR sensor.

\section{Results and Discussion}

When the RI of the cladding on the sensing ring changes, the envelope of the transmission spectrum of the cascaded double ring will shift. The radii of the reference ring and the sensing ring of the cascaded double-ring sensor are $123 \mu \mathrm{m}$ and $121 \mu \mathrm{m}$, respectively. When the RI of the sample increased, the transmission spectrum envelope of the cascaded double-ring sensor shifted to the short wavelength. Figure 6 showed the transmission spectra of the CDRR with the $\mathrm{NaCl}$ concentrations of $1.0 \%$ and $1.4 \%$. The envelope shift of the transmission spectrum can be measured by the Gaussian function fitting in the wavelength range of $8 \mathrm{~nm}$. The difference between $1.0 \%$ and $1.4 \%$ of the sol-

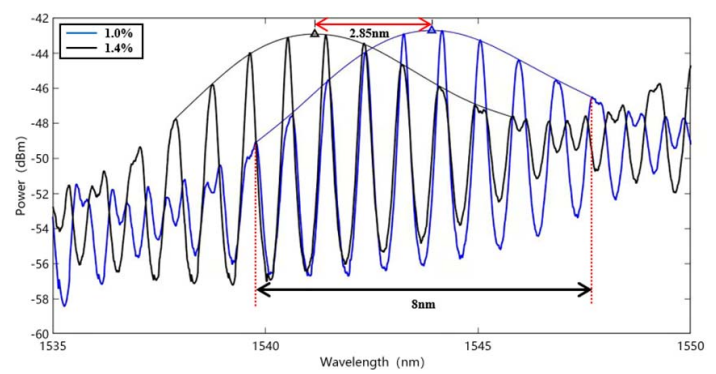

Fig. 6. Transmission spectra of drop port with $1.0 \% \mathrm{NaCl}$ solution and $1.4 \% \mathrm{NaCl}$ solution.

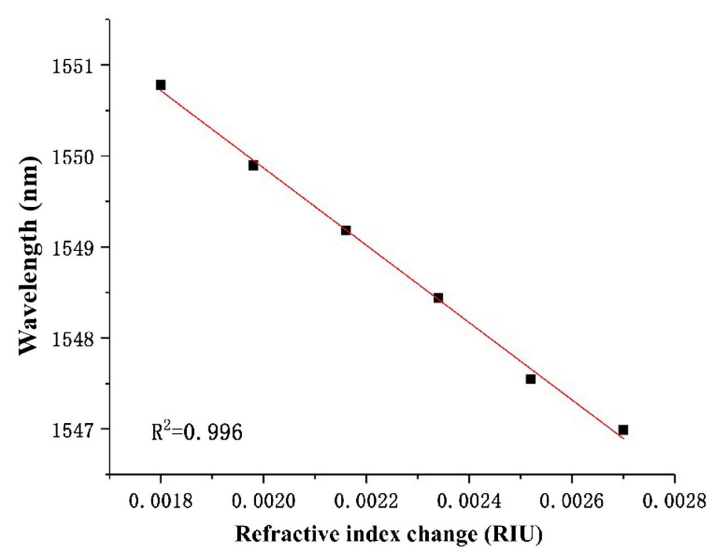

Fig. 7. Wavelength shift versus the RI change. 


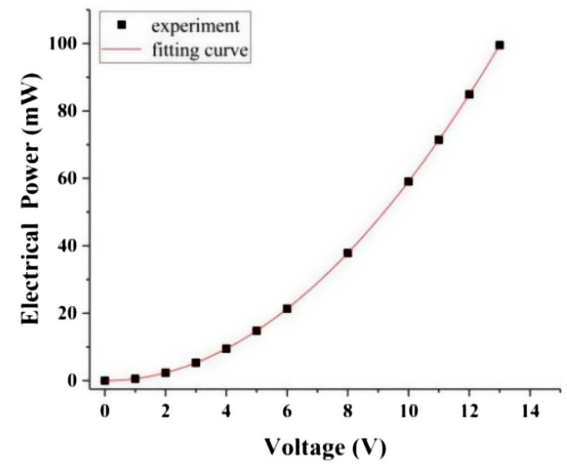

Fig. 8. Peak-to-valley (P-V) curve of the micro-heater.

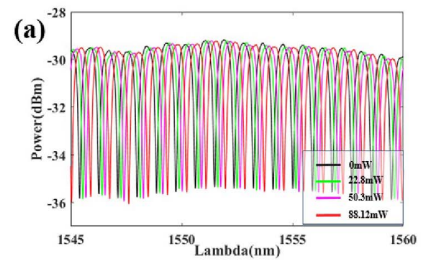

(c)
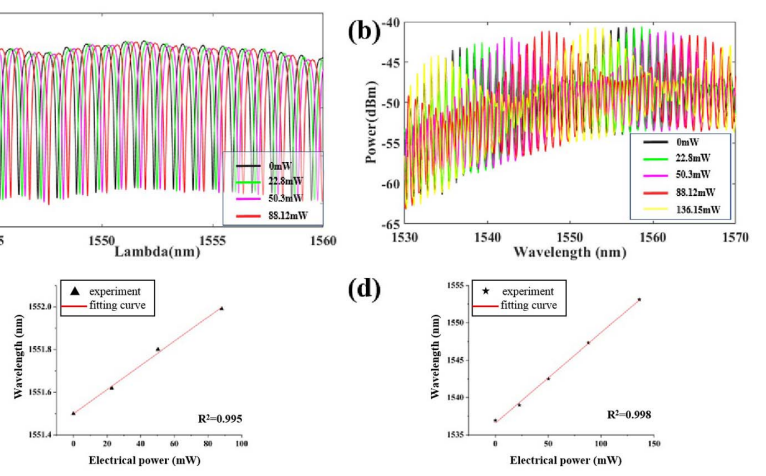

(d)

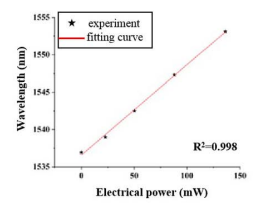

Fig. 9. Comparison of thermal tuning coefficients of single ring and double ring.

ution concentration resulted in a blue envelope peak shift of $2.85 \mathrm{~nm}$, as shown in Fig. 6.

By using different concentrations $(1.0 \%, 1.1 \%, 1.2 \%, 1.3 \%$, $1.4 \%$, and $1.5 \%$ ) of $\mathrm{NaCl}$ solution, the $\mathrm{RI}$ sensitivity without a thermal-optical tuning CDRR sensor was calibrated. For every $1 \%$ change in concentration, the RI changes $1.8 \times 10^{-3}$. As shown in Fig. 7, the measured RI sensitivity is $4244.44 \mathrm{~nm} / \mathrm{RIU}$.
The resistance of the micro-heater was above $1.8 \mathrm{k} \Omega$, as shown in Fig. 8, by fitting the curve of voltage versus electrical power.

The transmission spectra of the reference ring through the port and the CDRR with the different electrical powers $(0 \mathrm{~mW}, 22.8 \mathrm{~mW}, 50.3 \mathrm{~mW}, 88.12 \mathrm{~mW}$, and $136.15 \mathrm{~mW})$ are shown in Figs. 9(a) and 9(b), respectively. With the electric power increasing, the envelope peak results in a spectral redshift in Fig. 9(b). The measured shift of the single-ring resonant wavelength and the CDRR transmission spectrum envelope peak as a function of the electrical power are shown in Figs. 9(c) and $9(\mathrm{~d})$, respectively. According to the linear fitting, the thermal tuning coefficients of the single-ring and CDRR were $0.006 \mathrm{~nm} / \mathrm{mW}$ and $0.121 \mathrm{~nm} / \mathrm{mW}$, respectively. The results showed that the Vernier effect increased the thermal tuning coefficient by 20 times.

The sensitivity of the CDRR with the micro-heater for the thermal-optical tuning was measured in Fig. 10. The spectrum for the $\mathrm{NaCl}$ concentration of $1.0 \%$ is the initial spectrum. For the $\mathrm{NaCl}$ solutions of different concentrations $(1.1 \%, 1.2 \%, 1.3 \%$, $1.4 \%$, and $1.5 \%$ ), the output spectrum envelope was retrieved into the initial spectrum by changing the electric power of the micro-heater. The transmission spectrum was fitted by Gaussian function only in the $8 \mathrm{~nm}$ wavelength range.

The linear fitting relationship between the retrieved electrical power and the RI change is shown in Fig. 10(b). The thermooptical tuning sensitivity of the CDRR is $33.703 \mathrm{~W} / \mathrm{RIU}$. The limit of detection (LOD) can be expressed as $3 \sigma / S$. The $\sigma$ is obtained by the root mean square of the retrieved electric power in four times measurement, $\sigma=0.15 \mathrm{~mW}$. The LOD is $1.34 \times 10^{-5}$ RIU.

\section{Conclusion}

An optical sensing system based on the thermo-optical effect of CDRR is proposed in this paper. The sensing system included a sensing ring and a reference ring with a micro-heater for thermo-optical tuning. The retrieved electrical power was
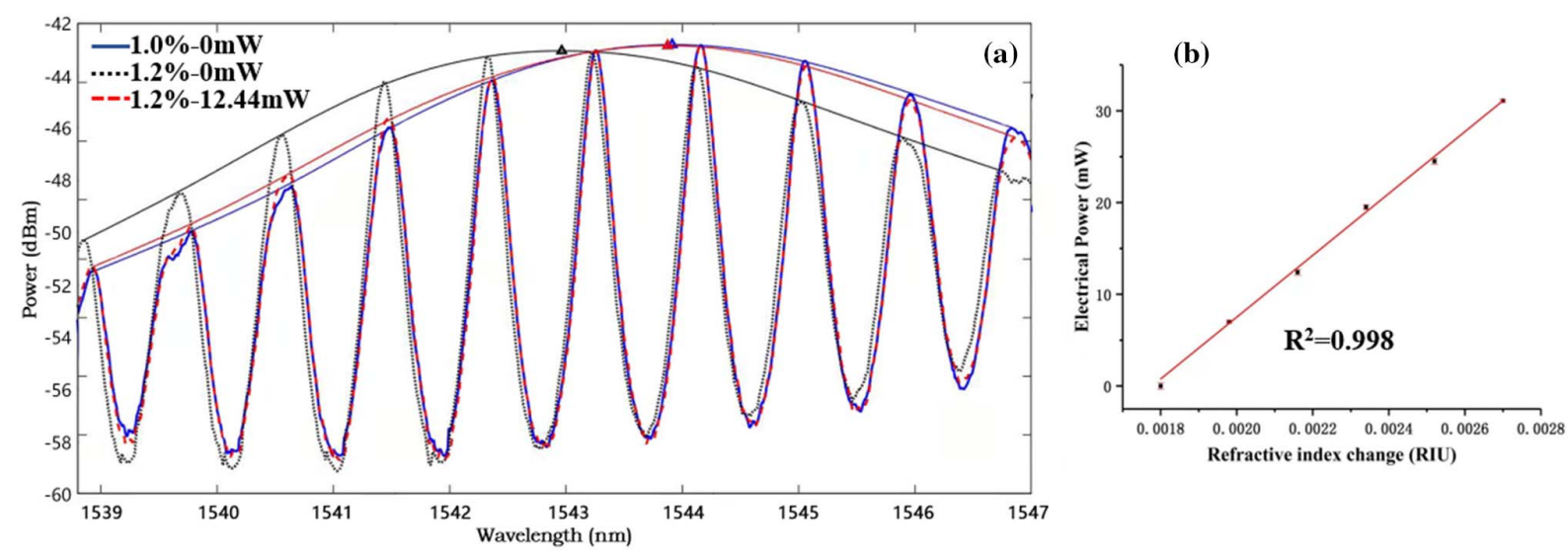

Fig. 10. (a) Retrieved spectra for $1.2 \%$ concentration of $\mathrm{NaCl}$ solution. (b) The linear fit curve of the thermo-optical tuning sensing sensitivity. 
measured for sensing the RI change of the sample. The sensing system did not require a broad bandwidth spectrometer or a large range wavelength tunable laser. The envelopes of the transmission spectra were retrieved by fitting the Gauss function in the $8 \mathrm{~nm}$ wavelength range. The sensitivity for the TM mode was $33.703 \mathrm{~W} / \mathrm{RIU}$, and the LOD was $1.34 \times 10^{-5} \mathrm{RIU}$.

\section{Acknowledgement}

This work was supported by the National Key Research and Development Program of China (No. 2020YFC2004600), the National Natural Science Foundation of China (No. 62027825), and the Research Foundation of the Education Department of Jilin province (No. JJKH20210822KJ). The authors acknowledge the wafer preparation and technical assistance by the Integrated Circuit Advanced Process Center (ICAC) of Institute of Microelectronics of Chinese Academy of Sciences (IMECAS).

\section{References}

1. L. Qing, W. K. Kyung, K. Jack, J. Song, and K. P. Mi, “Thermal characterization of electrical tracing-assisted dual-microring optical sensors," Opt. Express 22, 27069 (2014).

2. J. Song, X. Luo, S. K. Jack, H. Kyung, C. Li, K. P. Mi, X. Tu, X. Zhang, Q. Fang, L. Jia, J. Y. Yong, Y. L. Tsung, M. Yu, and G. Luo, "Silicon-based optoelectronic integrated circuit for label-free bio/chemical sensor," Opt. Express 21, 17931 (2013).

3. J. Song, X. Tu, M. K. Park, J. S. Kee, H. Zhang, M. Yu, G. Luo, and D. L. Kwong, "Electrical tracing assisted dual microring label free optical bio/chemical sensors," Opt. Express 20, 4189 (2012).

4. R. P. Prashanth, K. S. Shankar, and M. V. Manoj, "Full-range detection in cascaded microring sensors using thermooptical tuning," J. Lightwave Technol. 34, 5157 (2016).

5. G.-D. Kim, H.-S. Lee, C.-H. Park, S.-S. Lee, B. T. Lim, H. K. Bae, and W.-G. Lee, "Silicon photonic temperature sensor employing a ring resonator manufactured using a standard CMOS process," Opt. Express 18, 22215 (2010).
6. S. Li, R. Cong, Z. He, T. Wang, F. Zhang, and S. Pan, "Switchable microwave photonic filter using a phase modulator and a silicon-on-insulator microring resonator," Chin. Opt. Lett. 18, 052501 (2020).

7. Y.-P. Qi, X.-W. Zhang, P.-Y. Zhou, B.-B. Hu, and X.-X. Wang, "Refractive index sensor and filter of metal-insulator-metal waveguide based on ring resonator embedded by cross structure,” Acta Phys. Sin. 67, 197301 (2018).

8. X.-W. Dong, L. Pei, and S.-S. Jian, “Transfer matrix method for analyzing the characteristics of multiple-ring higher order microring resonators," Chin. J. Lasers 32, 929 (2005).

9. F. Meng, H. Yu, X. Zhou, Y. Li, M. Wang, W. Yang, W. Chen, Y. Zhang, and J. Pan, "Quantum wells micro-ring resonator laser emitting at $1746 \mathrm{~nm}$ for gas sensing," Chin. Opt. Lett. 19, 041406 (2021).

10. F. Y. Meng, H. Y. Yu, X. L. Zhou, Y. J. Li, M. Q. Wang, W. Y. Yang, W. X. Chen, Y. J. Zhang, and J. Q. Pan, "Quantum wells micro-ring resonator laser emitting at $1746 \mathrm{~nm}$ for gas sensing," Chin. Opt. Lett. 19, 041406 (2021).

11. W.-Y. Deng, S.-L. E, C.-S. Ma, H.-D. Zhao, and W. Xu, "Multi channel access micro ring resonator wavelength selective switching characteristics," Acta Photon. Sin. 37, 2394 (2008).

12. X. Tong, K. Han, X.-P. Shen, Q.-H. Wu, F. Zhou, Y. Ge, and X.-J. Hu, "Equal intensity polarization-independent beam splitter based on photonic crystal self-collimation ring resonator,” Acta Phys. Sin. 60, 064217 (2011).

13. M. You, Z. Lin, X. Li, and J. Liu, "Chip-scale silicon ring resonators for cryogenic temperature sensing," J. Lightwave Technol. 38, 5768 (2020).

14. J. Song, X. Luo, X. Tu, K. P. Mi, S. K. Jack, H. Zhang, M. Yu, G. Luo, and D. L. Kwong, "Electrical tracing-assisted dual-microring label-free optical bio/chemical sensors," Opt. Express 20, 4189 (2012).

15. Y. Liu, Y. Li, M. Li, and J. J. He, "High-sensitivity and wide-range optical sensor based on three cascaded ring resonators," Opt. Express 25, 972 (2017).

16. Y. Zhang, J. Zou, Z. Cao, and J. J. He, “Temperature insensitive waveguide sensor using a ring cascaded with a Mach Zehnder interferometer," Opt. Lett. 44, 299 (2019).

17. Y. Yue, H. Zhu, Z. Cao, J. He, and M. Li, "Wide-range optical sensors based on a single ring resonator with polarization multiplexing," Chin. Opt. Lett. 17, 031301 (2019).

18. X. Jiang, L. Tang, J. Song, M. Li, and J.-J. He, “Optical waveguide biosensor based on cascaded Mach-Zehnder interferometer and ring resonator with Vernier effect," Proc. SPIE 9310, 931003 (2015).

19. L. Jin, M. Li, and J. J. He, "Highly sensitive silicon on insulator sensor based on two cascaded micro ring resonators with Vernier effect," Opt. Commun. 284, 156 (2011).

20. C. Su, H.-H. Zhu, Z.-W. Cao, J.-J. He, and M.-Y. Li, "Fitting methods of transmission spectrum envelope in cascaded double-ring resonator sensors," Acta Photon. Sin. 47, 1023002 (2018). 